\title{
A FRAMEWORK FOR PRUNING DEEP NEURAL NETWORKS USING ENERGY-BASED MODELS
}

\author{
Hojjat Salehinejad, Member, IEEE, and Shahrokh Valaee, Fellow, IEEE \\ Department of Electrical \& Computer Engineering, University of Toronto, Toronto, Canada \\ hojjat.salehinejad@mail.utoronto.ca,valaee@ece.utoronto.ca
}

\begin{abstract}
A typical deep neural network (DNN) has a large number of trainable parameters. Choosing a network with proper capacity is challenging and generally a larger network with excessive capacity is trained. Pruning is an established approach to reducing the number of parameters in a DNN. In this paper, we propose a framework for pruning DNNs based on a population-based global optimization method. This framework can use any pruning objective function. As a case study, we propose a simple but efficient objective function based on the concept of energy-based models. Our experiments on ResNets, AlexNet, and SqueezeNet for the CIFAR-10 and CIFAR-100 datasets show a pruning rate of more than $50 \%$ of the trainable parameters with approximately $<5 \%$ and $<1 \%$ drop of Top-1 and Top-5 classification accuracy, respectively.
\end{abstract}

Index Terms - Compression of neural networks, dropout, energy-based models, pruning.

\section{INTRODUCTION}

Pruning a deep neural network (DNN) is one of the major methods for removing redundant trainable parameters and compressing the network. This approach permanently removes a subset of trainable parameters. In general, pruning algorithms have three stages which are training, pruning, and fine-tuning [1]. One pruning approach is to utilize second derivative information to minimize a cost function that reduces network complexity by removing excess number of trainable parameters and further fine-tuning [2]. One of the major approaches is Deep Compression, which has three main steps that are pruning, quantization, and Huffman coding [3]. It prunes all connections with weights below a given threshold and then retrains the sparsified network.

Generally, probabilistic models can be considered as a special type of energy-based models (EBMs). An EBM assigns a scalar energy loss as a measure of compatibility to a configuration of parameters in neural networks as demonstrated in Figure 1 This approach avoids computing the normalization term, which can be interpreted as an alternative to probabilistic estimation [4]. Calculating the exact probability needs computing the partition function over all the data

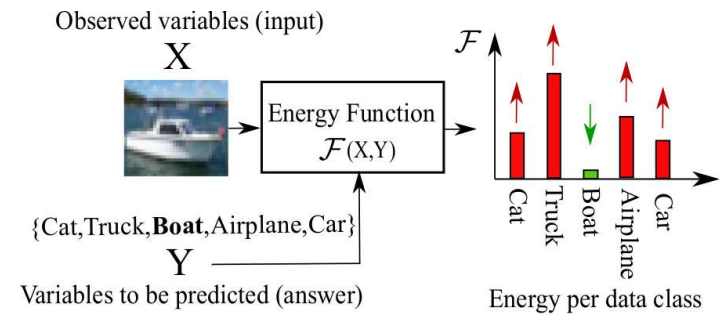

Fig. 1: Energy-based model (EBM), 4].

classes. However, for large number of data classes, such as in language models with more than 100, 000 classes, this causes a bottleneck [5]. Some methods such as annealed importance sampling [6] have been proposed to deal with this problem which is out of the scope of this paper.

Previously, we have proposed an Ising energy model for dropout and pruning of multilayer perceptron (MLP) networks [7, 8]. In this paper, a pruning framework based on a population-based stochastic global optimization method is proposed which is integrated into the typical training procedure of a DNN. This scheme is inspired from the concept of dropout [9] and biological pruning of neurons in brain. This framework can handle different pruning objective function with multiple constrains. We also propose an energy-based pruning objective function based on the concept of EBM in DNN, which allocates a scalar energy value to each state vector in the population of state vectors, called EPruning. Each state vector is in fact a representation of a sub-network from the original DNN. Pruning is defined as searching for a binary state vector that prunes the network while minimizes the energy loss for a set of inputs and corresponding outputs in each iteration. Hence, the search for weights is conducted using a probabilistic model while the pruning state vector is fixed and the search for pruning state is conducted using an EBM while the weights are fixed in each iteration. The candidate states help to find a subset of the neural network and capture its energy function that associates low energies to correct values of the remaining variables, and higher energies to incorrect value: 1 .

\footnotetext{
${ }^{1}$ The codes and more details of experiments setup is available at: https://github.com/sparsifai/epruning
} 


\section{PROPOSED PRUNING FRAMEWORK}

\subsection{Energy Model}

A DNN can be modeled as a parametric function to map the input image $X \in \mathcal{X}$ to $C$ real-valued numbers $\epsilon=$ $\left\{\varepsilon_{1}, \varepsilon_{2}, \ldots, \varepsilon_{C}\right\}$ (a.k.a. logits). The output is then passed to a classifier, such as Softmax function to parameterize a categorical distribution in form of a probability distribution over the data classes $\mathcal{Y}=\left\{y_{1}, \ldots, y_{C}\right\}$ [10], defined as $\left\{p\left(y_{1}\right), \ldots, p\left(y_{C}\right)\right\}$ where for simplicity we define $p_{c}=p\left(y_{c}\right) \forall c \in\{1, \ldots, C\}$, as illustrated in Figure 2. The loss is then calculated based on cross-entropy with respect to the correct answer $Y$. Gibbs distribution is a very general family of probability distributions defined as

$$
p(Y \mid X)=\frac{e^{-\beta \mathcal{F}(Y, X)}}{Z(\beta)},
$$

where $Z(\beta)=\sum_{y_{c} \in \mathcal{Y}} e^{-\beta \mathcal{F}\left(y_{c}, X\right)}$ is the partition function, $\beta>0$ is the inverse temperature parameter [4], and $\mathcal{F}(\cdot)$ is the Hamiltonian or the energy function. Softmax function is a special case of the Gibbs distribution. We can achieve the energy function corresponding to using a Softmax layer by setting $\beta=1$ [11] in (1) and getting the Hamiltonian

$$
\mathcal{F}(Y, X)=-\epsilon \text {. }
$$

We define the following energy loss function to measure the quality of energy function for $(X, Y)$ with target output $y_{c}$ as

$$
\begin{aligned}
\mathcal{E} & =\mathcal{L}(Y, \mathcal{F}) \\
& =\mathcal{F}\left(y_{c}, X\right)-\min \left\{\mathcal{F}\left(y_{c^{\prime}}, X\right): y_{c^{\prime}} \in \mathcal{Y}, c^{\prime} \neq c\right\},
\end{aligned}
$$

where it can be extended for a batch of data. The energy loss function assigns a low loss value to a pruning state vector which has the lowest energy with respect to the target data class $c$ and higher energy with respect to the other data classes and vice versa [4].

\subsection{Training and Optimization}

We are interested in pruning weight kernels and hidden units, including their bias terms, referred to as a unit hereafter for convenience. We define a set of $S$ binary candidate pruning state vectors as the population $\mathbf{S}^{S \times D}$. Each vector $\mathbf{s}_{i} \in \mathbf{S}$ has a length of $D$ which represents a sub-network. The energy function value for each state vector is $\mathcal{F}_{i} \in\left\{\mathcal{F}_{1}, \ldots, \mathcal{F}_{S}\right\}$. If $s_{i, d}=0$ unit $d$ is dropped and if $s_{i, d}=1$ it is active.

Algorithm 1 shows different steps of the proposed framework. At the beginning of training $(t=0)$, we initialize the candidate pruning states $\mathbf{S}^{(0)} \in \mathbb{Z}_{2}^{S \times D}$, where $s_{i, d}^{(0)} \sim$ Bernoulli $(P=0.5)$ for $i \in\{1, \ldots, S\}$ and $d \in\{1, \ldots, D\}$.

For each candidate state $\mathbf{s}_{i}^{(t)} \in \mathbf{S}^{(t)}$ in iteration $t$, the energy loss value is calculated using (3) as $\mathcal{E}_{i}^{(t)}$. Searching for the pruning state which can minimize the energy loss

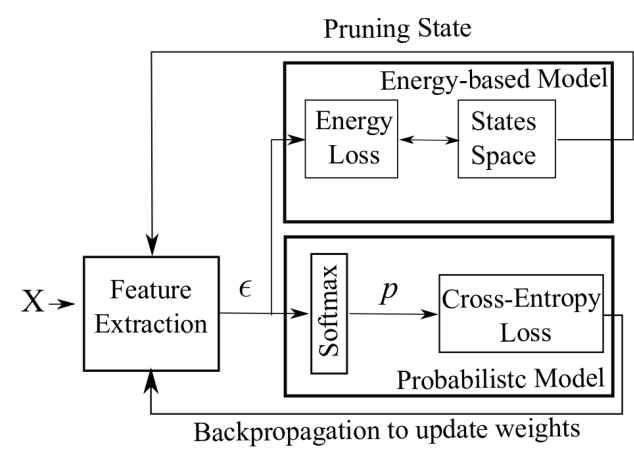

Fig. 2: Switching between the energy-based model (EBM) and the probabilistic model. The EBM searches for the pruning state and the probabilistic models searches for the weights. Both models are aware of target class $Y$ during training. In inference, the best pruning state is applied and the EBM is removed.

value is an NP-hard combinatorial problem. Various methods such as MCMC [12] and simulated annealing (SA) can be used to search for low energy states. We propose using a binary version of differential evolution (BDE) [13] to minimize the energy loss function. This method has the advantage of searching the optimization landscape in parallel and sharing the search experience among candidate states. The other advantage of this approach is flexibility of designing the energy function with constraints.

The optimization step has three phases which are mutation, crossover, and selection. Given the population of states $\mathbf{S}^{(t-1)}$, a mutation vector is defined for each candidate state $\mathbf{s}_{i}^{(t-1)} \in \mathbf{S}^{(t-1)}$ as

$$
v_{i, d}= \begin{cases}1-s_{i_{1}, d}^{(t-1)}, & \text { if } s_{i_{2}, d}^{(t-1)} \neq s_{i_{3}, d}^{(t-1)} \& r_{d}<F \\ s_{i_{1}, d}^{(t-1)}, & \text { otherwise }\end{cases}
$$

for all $d \in\{1, . ., D\}$, where $i_{1}, i_{2}, i_{3} \in\{1, \ldots, S\}$ are mutually different, $F$ is the mutation factor [14], and $r_{d} \in[0,1]$ is a random number. The next step is to crossover the mutation vectors to generate new candidate state vectors as

$$
\tilde{s}_{i, d}^{(t)}=\left\{\begin{array}{lc}
v_{i, d} & \text { if } r_{d}^{\prime} \in[0,1] \leq C_{r} \\
s_{i, d}^{(t-1)} & \text { otherwise }
\end{array},\right.
$$

where $C_{r}$ is the crossover coefficient [14]. The parameters $C_{r}$ and $F$ control exploration and exploitation of the population on the optimization landscape. Each generated state $\tilde{\mathbf{s}}_{i}^{(t)}$ is then compared with its corresponding parent with respect to its energy loss value $\tilde{\mathcal{E}}_{i}^{(t)}$ as

$$
\mathbf{s}_{i}^{(t)}=\left\{\begin{array}{ll}
\tilde{\mathbf{s}}_{i}^{(t)} & \text { if } \tilde{\mathcal{E}}_{i}^{(t)} \leq \mathcal{E}_{i}^{(t-1)} \\
\mathbf{s}_{i}^{(t-1)} & \text { otherwise }
\end{array} \quad \forall i \in\{1, \ldots, S\}\right.
$$

The state with minimum energy loss $\mathcal{E}_{b}^{(t)}=\min \left\{\mathcal{E}_{1}^{(t)}, \ldots, \mathcal{E}_{S}^{(t)}\right\}$ is selected as the best state $\mathbf{s}_{b}$, which represents the subnetwork for next training batch. This optimization strategy is simple and feasible to implement in parallel for a large $S$. 
The population-based optimization methods suffer from premature convergence and stagnation problems. The former generally occurs when the population (candidate state vectors) has converged to local optima, has lost its diversity, or has no improvement in finding better solutions. The latter happens mainly when the population stays diverse during training [15]. After a number of iterations, depending on the capacity of the neural network and the complexity of the dataset, all the states in $\mathbf{S}^{(t)}$ may converge to a state $\mathbf{s}_{b} \in \mathbf{S}^{(t)}$. We call this the early state convergence phase and define it as

$$
\Delta \mathbf{s}=\mathcal{E}_{b}^{(t)}-\frac{1}{S} \sum_{j=1}^{S} \mathcal{E}_{j}^{(t)},
$$

where $\mathcal{E}_{b}^{(t)}$ is the energy loss of $\mathbf{s}_{b}$. Therefore, if $\Delta \mathbf{s}=0$ we can call for an early state convergence and continue training by fine-tuning the sub-network identified by the state vector $\mathbf{s}_{b}$. In addition, a stagnation threshold $\Delta \mathbf{s}_{T}$ is implemented where if $\Delta \mathbf{s} \neq 0$ after $\Delta \mathbf{s}_{T}$ number of training epochs, it stops the energy loss optimizer and starts fine-tuning the selected sub-network. The convergence to the best state $\mathbf{s}_{b}$ splits the training procedure into two phases, where the first phase acts similar to dropout and the second phase fine-tunes the pruned network, defined by $\mathbf{s}_{b}$.

\section{EXPERIMENTS}

The experiments were conducted on the CIFAR-10 and CIFAR-100 [16] datasets. The horizontal flip and Cutout [17] augmentation methods were used. Input images were resized to $32 \times 32$ for ResNets and $224 \times 224$ for AlexNet [18] and SqueezeNet v1.1 [19].

We used ResNets (18, 34, 50, and 101 layers) [20], AlexNet [18], SqueezeNet v1.1 [19], and Deep Compression [3] to evaluate EPruning. The results were averaged over five independent runs. A grid hyper-parameter search was conducted based on the Top-1 accuracy for all models, including initial learning rates in $\{0.01,0.1,1\}$, Stochastic Gradient Descent (SGD) [21] and Adadelta [22] optimizer, exponential and step learning rate decays with gamma values in $\{25,50\}$, and batch sizes of 64 and 128. The Adadelta optimizer with Step adaptive learning rate (step: every 50 epoch at gamma rate of 0.1 ) and weight decay of $10 e^{-6}$ was used. The number of epochs was 200 and the batch size was 128 . Random dropout was not used in the EPruning experiments. For the other models, where applicable, the random dropout rate was set to 0.5 . The early state convergence in (7) is used with a threshold of 100 epochs. The models are implemented in PyTorch [23] and trained on three NVIDIA Titan RTX GPUs.

Table 1 shows the classification performance results. The original models contain the entire trainable parameters and have larger learning capacity. EPruning in pruned and full

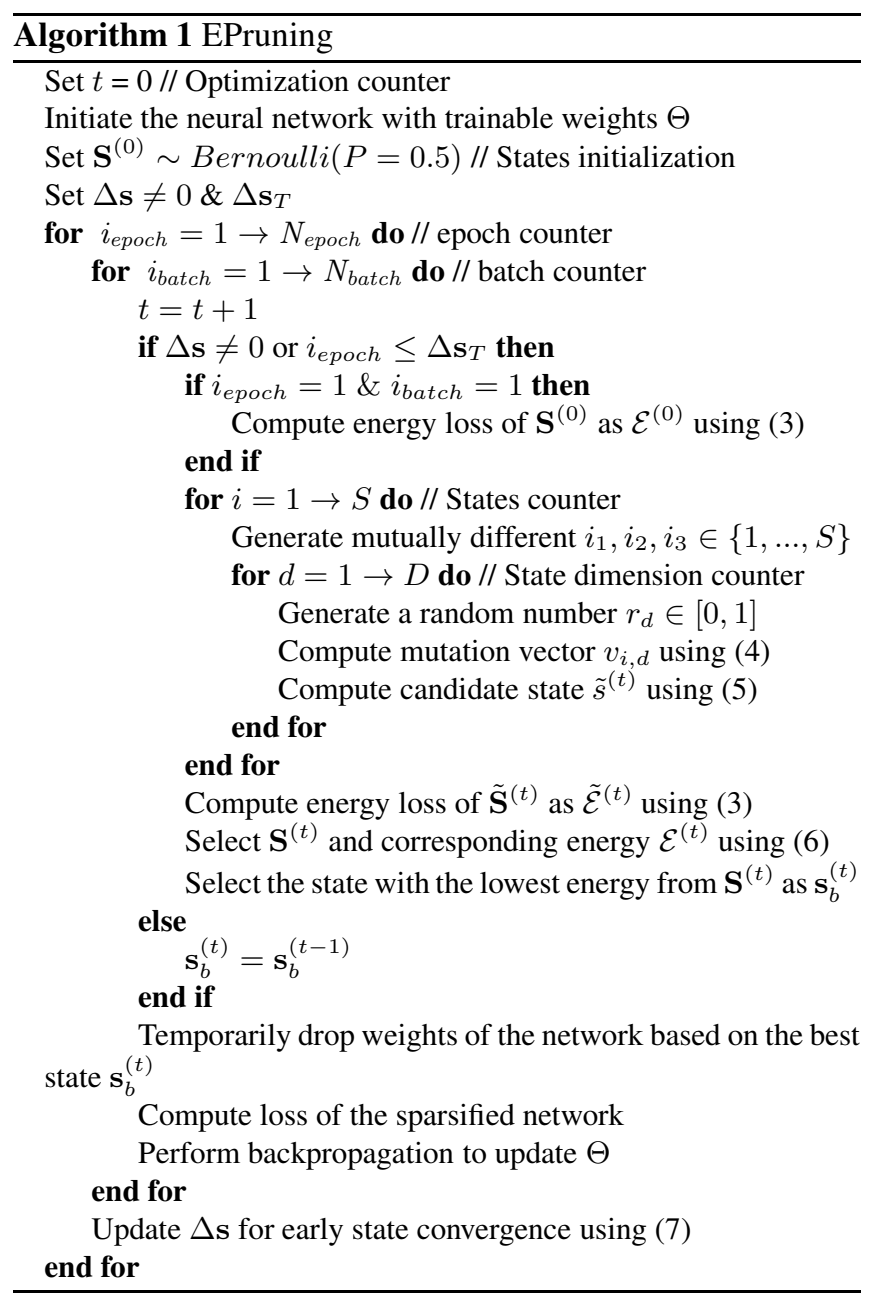

versions have slightly lower Top-1 performance than the original model and competitive performance in terms of Top-5 performance. The Deep Compression [3] method receives pruning rate as input. For the sake of comparison, we have modified it to perform pruning on every convolution layer, given the rate achieved by EPruning, where generally it has lower performance than EPruning. SqueezeNet [24] is a small network with AlexNet level accuracy. EPruning is also applied to AlexNet and SqueezeNet v1.1, where it has a smaller pruning rate for AlexNet compared to ResNets but can prune approximately half of the trainable parameters in SqueezeNet v1.1 and achieve slightly lower performance.

Figure 3 shows convergence plots of ResNet-50 with respect to the cross-entropy loss and energy of the sub-network over 200 training epochs. The plots show that the best energy is lower than the average energy and after epoch 100, due to early stopping, the sub-network is selected. Training loss and energy loss follow similar declining trend and after epoch 100 , the training loss has a slower declining rate. We observe that before epoch 100 the model was in an exploration phase and after this epoch enters an exploitation (fine-tuning) phase. 


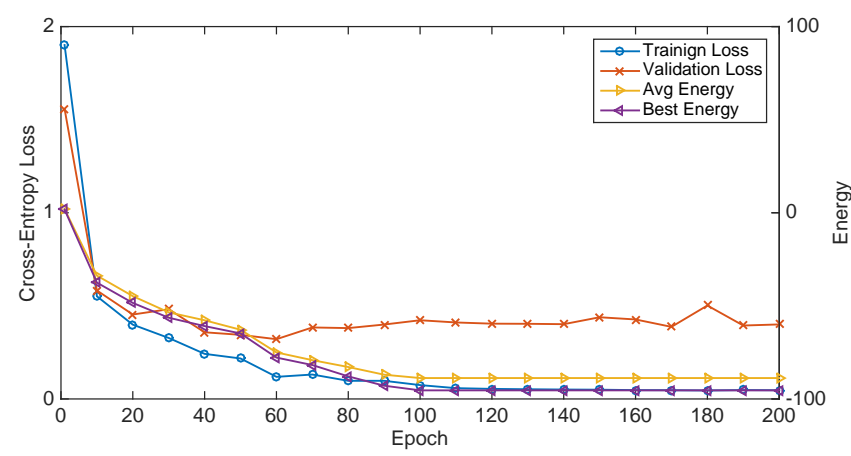

(a) CIFAR-10

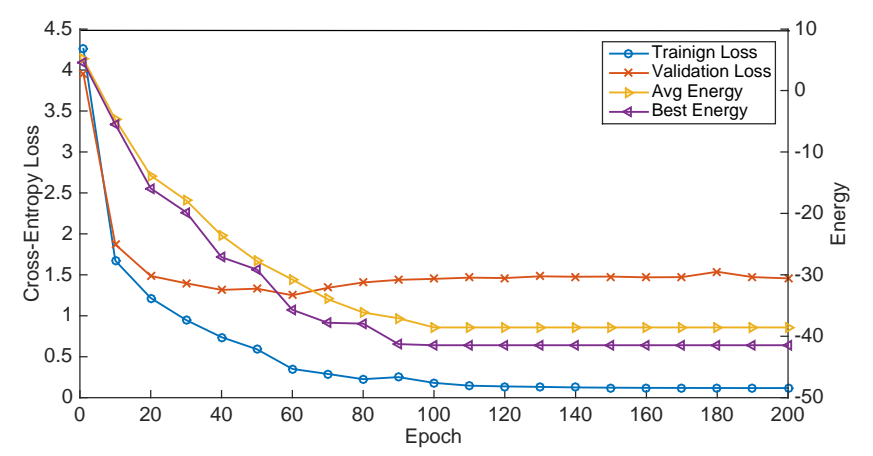

(b) CIFAR-100

Fig. 3: Cross-Entropy loss and energy of EPruning over 200 training epochs of ResNet-50 for CIFAR-10 and CIFAR-100 datasets with $S=8$, initialization probability of $P=0.5$, and $\Delta \mathbf{s}_{T}=100$.

Table 1: Classification performance on test datasets. $R$ is kept trainable parameters and \#p is approximate number of trainable parameters. All the values except loss and \#p are in percentage. (F) refers to full network used for inference and (P) refers to pruned network using EPruning.

(a) CIFAR-10

\begin{tabular}{lllllll}
\hline \multicolumn{1}{c}{ Model } & Loss & Top-1 & Top-3 & Top-5 & \multicolumn{1}{c}{$R$} & $\# p$ \\
\hline \hline ResNet-18 & 0.3181 & 92.81 & 98.78 & 99.49 & 100 & $11.2 \mathrm{M}$ \\
ResNet-18+DeepCompression & 0.6951 & 76.15 & 94.16 & 98.59 & 49.66 & $5.5 \mathrm{M}$ \\
ResNet-18+EPruning(F) & 0.4906 & 90.96 & 98.33 & 99.60 & 100 & $11.2 \mathrm{M}$ \\
ResNet-18+EPruning(P) & 0.4745 & 90.96 & 98.40 & 99.58 & 49.66 & $5.5 \mathrm{M}$ \\
\hline \hline ResNet-34 & 0.3684 & 92.80 & 98.85 & 99.71 & 100 & $21.3 \mathrm{M}$ \\
ResNet-34+DeepCompression & 1.057 & 66.51 & 91.40 & 97.68 & 38.83 & $8.3 \mathrm{M}$ \\
ResNet-34+EDropou(F) & 0.4576 & 88.28 & 97.47 & 99.31 & 100 & $21.3 \mathrm{M}$ \\
ResNet-34+EPruning(P) & 0.4598 & 88.21 & 97.48 & 99.28 & 38.83 & $8.3 \mathrm{M}$ \\
\hline \hline ResNet-50 & 0.3761 & 92.21 & 98.70 & 99.51 & 100 & $23.5 \mathrm{M}$ \\
ResNet-50+DeepCompression & 1.0271 & 67.53 & 89.92 & 96.30 & 46.39 & $10.9 \mathrm{M}$ \\
ResNet-50+EPruning(F) & 0.6041 & 85.22 & 96.35 & 98.77 & 100 & $23.5 \mathrm{M}$ \\
ResNet-50+EPruning(P) & 0.5953 & 85.30 & 96.62 & 98.76 & 46.39 & $10.9 \mathrm{M}$ \\
\hline \hline ResNet-101 & 0.3680 & 92.66 & 98.69 & 99.65 & 100 & $42.5 \mathrm{M}$ \\
ResNet-101+DeepCompression & 1.037 & 66.32 & 92.65 & 98.11 & 45.10 & $19.2 \mathrm{M}$ \\
ResNet-101+EPruning(F) & 0.6231 & 86.97 & 97.42 & 99.24 & 100 & $42.5 \mathrm{M}$ \\
ResNet-101+EPruning(P) & 0.6339 & 86.57 & 97.37 & 99.20 & 45.10 & $19.2 \mathrm{M}$ \\
\hline \hline AlexNet & 0.9727 & 84.32 & 96.58 & 99.08 & 100 & $57.4 \mathrm{M}$ \\
AlexNet+EPruning(F) & 0.7632 & 75.05 & 93.74 & 98.18 & 100 & $57.4 \mathrm{M}$ \\
AlexNet+EPruning(P) & 0.7897 & 74.66 & 93.63 & 97.96 & 77.36 & $44.4 \mathrm{M}$ \\
SqueezeNet & 0.5585 & 81.49 & 96.31 & 99.01 & 100 & $0.73 \mathrm{M}$ \\
SqueezeNet+EPruning(F) & 0.6686 & 76.76 & 94.55 & 98.62 & 100 & $0.73 \mathrm{M}$ \\
SqueezeNet+EPruning(P) & 0.6725 & 76.85 & 95.00 & 98.56 & 52.35 & $0.38 \mathrm{M}$ \\
\hline
\end{tabular}

This method increases the computational complexity of the model due to evaluation of energy loss for each candidate state vector in the population. However, with proper parallel implementation at each state vector level and shared memory management, this overhead can significantly be reduced. As an example, for ResNet-50, the inference time per image is 5.50E-5 Seconds where with EPruning with a population size of 8 this time is $3.40 \mathrm{E}-4$ Seconds and in parallel mode this time is $8.95 \mathrm{E}-5$ Seconds.

\section{CONCLUSIONS}

In this paper, we have proposed a stochastic framework for pruning deep neural networks. Most pruning and compression models first prune a network and then fine-tune it. The proposed EPruning method has two phases. The first phase acts as dropout where various subsets of the neural network (b) CIFAR-100

\begin{tabular}{lcccccc}
\hline \multicolumn{1}{c}{ Model } & Loss & Top-1 & Top-3 & Top-5 & $R$ & $\# p$ \\
\hline \hline ResNet-18 & 1.3830 & 69.03 & 84.44 & 88.90 & 100 & $11.2 \mathrm{M}$ \\
ResNet-18+DeepCompression & 2.3072 & 40.01 & 62.20 & 72.28 & 48.04 & $5.4 \mathrm{M}$ \\
ResNet-18+EPruning(F) & 1.9479 & 67.04 & 84.11 & 89.43 & 100 & $11.2 \mathrm{M}$ \\
ResNet-18+EPruning(P) & 1.9541 & 67.06 & 84.14 & 89.27 & 48.04 & $5.4 \mathrm{M}$ \\
\hline \hline ResNet-34 & 1.3931 & 69.96 & 85.65 & 90.10 & 100 & $21.3 \mathrm{M}$ \\
ResNet-34+DeepCompression & 2.1778 & 42.09 & 65.01 & 74.31 & 49.41 & $10.5 \mathrm{M}$ \\
ResNet-34+EPruning(F) & 1.9051 & 64.50 & 81.38 & 86.87 & 100 & $21.3 \mathrm{M}$ \\
ResNet-34+EPruning(P) & 1.9219 & 64.79 & 81.28 & 86.74 & 49.41 & $10.5 \mathrm{M}$ \\
\hline \hline ResNet-50 & 1.3068 & 71.22 & 86.47 & 90.74 & 100 & $23.7 \mathrm{M}$ \\
ResNet-50+DeepCompression & 2.3115 & 43.87 & 67.02 & 76.26 & 46.01 & $10.9 \mathrm{M}$ \\
ResNet-50+EPruning(F) & 1.8750 & 61.60 & 79.52 & 85.45 & 100 & $23.7 \mathrm{M}$ \\
ResNet-50+EPruning(P) & 1.8768 & 61.91 & 79.99 & 85.87 & 46.01 & $10.9 \mathrm{M}$ \\
\hline \hline ResNet-101 & 1.3574 & 71.19 & 85.54 & 90.00 & 100 & $42.6 \mathrm{M}$ \\
ResNet-101+DeepCompression & 2.6003 & 37.08 & 58.78 & 68.76 & 43.76 & $18.6 \mathrm{M}$ \\
ResNet-101+EPruning(F) & 1.9558 & 61.52 & 79.71 & 85.20 & 100 & $42.6 \mathrm{M}$ \\
ResNet-101+EPruning(P) & 1.9412 & 61.92 & 79.49 & 85.23 & 43.76 & $18.6 \mathrm{M}$ \\
\hline \hline AlexNet & 2.8113 & 60.12 & 79.18 & 83.31 & 100 & $57.4 \mathrm{M}$ \\
AlexNet+EPruning(F) & 2.4731 & 56.62 & 78.72 & 81.92 & 100 & $57.4 \mathrm{M}$ \\
AlexNet+EPruning(P) & 2.4819 & 56.59 & 78.52 & 81.62 & 71.84 & $41.2 \mathrm{M}$ \\
SqueezeNet & 1.4150 & 67.85 & 85.81 & 89.69 & 100 & $0.77 \mathrm{M}$ \\
SqueezeNet+EPruning(F) & 1.5265 & 64.23 & 82.71 & 88.63 & 100 & $0.77 \mathrm{M}$ \\
SqueezeNet+EPruning(P) & 1.5341 & 64.02 & 81.63 & 88.51 & 56.40 & $0.43 \mathrm{M}$ \\
\hline
\end{tabular}

are trained. Each sub-network is selected based on a corresponding energy loss value which reflects performance of the sub-network. The second phase is a fine-tuning phase with focus on training the pruned network. The proposed framework has the advantage of immediate usability for any neural network without manual modification of layers. In addition, predefined number of active states can also be utilized in the optimizer to enforce a specific dropout/pruning rate. Our experiments show that as the proposed framework searches for sub-networks with lower energy, the training loss also decreases.

\section{ACKNOWLEDGMENT}

The authors acknowledge financial support of Fujitsu Laboratories Ltd. and Fujitsu Consulting (Canada) Inc. 


\section{REFERENCES}

[1] Zhuang Liu, Mingjie Sun, Tinghui Zhou, Gao Huang, and Trevor Darrell, "Rethinking the value of network pruning," arXiv preprint arXiv:1810.05270, 2018.

[2] Yann LeCun, John S Denker, and Sara A Solla, "Optimal brain damage," in Advances in neural information processing systems, 1990, pp. 598-605.

[3] Song Han, Huizi Mao, and William J Dally, "Deep compression: Compressing deep neural networks with pruning, trained quantization and huffman coding," arXiv preprint arXiv:1510.00149, 2015.

[4] Yann LeCun, Sumit Chopra, Raia Hadsell, M Ranzato, and F Huang, "A tutorial on energy-based learning," Predicting structured data, vol. 1, no. 0, 2006.

[5] David Barber and Aleksandar Botev, "Dealing with a large number of classes-likelihood, discrimination or ranking?," arXiv preprint arXiv:1606.06959, 2016.

[6] Radford M Neal, "Annealed importance sampling," Statistics and computing, vol. 11, no. 2, pp. 125-139, 2001.

[7] Hojjat Salehinejad and Shahrokh Valaee, "Isingdropout: A regularization method for training and compression of deep neural networks," in ICASSP 2019-2019 IEEE International Conference on Acoustics, Speech and Signal Processing (ICASSP). IEEE, 2019, pp. 3602-3606.

[8] Hojjat Salehinejad, Zijian Wang, and Shahrokh Valaee, "Ising dropout with node grouping for training and compression of deep neural networks," in 2019 IEEE Global Conference on Signal and Information Processing (GlobalSIP). IEEE, 2019, pp. 1-5.

[9] Alex Labach, Hojjat Salehinejad, and Shahrokh Valaee, "Survey of dropout methods for deep neural networks," arXiv preprint arXiv:1904.13310, 2019.

[10] Will Grathwohl, Kuan-Chieh Wang, Jörn-Henrik Jacobsen, David Duvenaud, Mohammad Norouzi, and Kevin Swersky, "Your classifier is secretly an energy based model and you should treat it like one," arXiv preprint arXiv:1912.03263, 2019.

[11] Kevin P Murphy, Machine learning: a probabilistic perspective, MIT press, 2012.

[12] Keivan Dabiri, Mehrdad Malekmohammadi, Ali Sheikholeslami, and Hirotaka Tamura, "Replica exchange mcmc hardware with automatic temperature selection and parallel trial," IEEE Transactions on Parallel and Distributed Systems, vol. 31, no. 7, pp. 1681-1692, 2020.
[13] Kenneth V Price, “Differential evolution,” pp. 187-214, 2013.

[14] Hojjat Salehinejad, Shahryar Rahnamayan, and Hamid R Tizhoosh, "Micro-differential evolution: Diversity enhancement and a comparative study," Applied Soft Computing, vol. 52, pp. 812-833, 2017.

[15] Jouni Lampinen, Ivan Zelinka, et al., "On stagnation of the differential evolution algorithm," in Proceedings of MENDEL, 2000, pp. 76-83.

[16] Alex Krizhevsky, Geoffrey Hinton, et al., "Learning multiple layers of features from tiny images," 2009.

[17] Terrance DeVries and Graham W Taylor, "Improved regularization of convolutional neural networks with cutout," arXiv preprint arXiv:1708.04552, 2017.

[18] Alex Krizhevsky, Ilya Sutskever, and Geoffrey E Hinton, "Imagenet classification with deep convolutional neural networks," in Advances in neural information processing systems, 2012, pp. 1097-1105.

[19] Forrest N Iandola, Song Han, Matthew W Moskewicz, Khalid Ashraf, William J Dally, and Kurt Keutzer, "Squeezenet: Alexnet-level accuracy with 50x fewer parameters andi $0.5 \mathrm{mb}$ model size," arXiv preprint arXiv:1602.07360, 2016.

[20] Kaiming He, Xiangyu Zhang, Shaoqing Ren, and Jian Sun, "Deep residual learning for image recognition," in Proceedings of the IEEE conference on computer vision and pattern recognition, 2016, pp. 770-778.

[21] Geoffrey Hinton, Nitish Srivastava, and Kevin Swersky, "Neural networks for machine learning lecture $6 a$ overview of mini-batch gradient descent," Cited on, vol. 14, no. 8, 2012.

[22] Matthew D Zeiler, "Adadelta: an adaptive learning rate method," arXiv preprint arXiv:1212.5701, 2012.

[23] Adam Paszke, Sam Gross, Francisco Massa, Adam Lerer, James Bradbury, Gregory Chanan, Trevor Killeen, Zeming Lin, Natalia Gimelshein, Luca Antiga, Alban Desmaison, Andreas Kopf, Edward Yang, Zachary DeVito, Martin Raison, Alykhan Tejani, Sasank Chilamkurthy, Benoit Steiner, Lu Fang, Junjie Bai, and Soumith Chintala, "Pytorch: An imperative style, high-performance deep learning library," in Advances in Neural Information Processing Systems 32, pp. 8024-8035. Curran Associates, Inc., 2019.

[24] Forrest N Iandola, Song Han, Matthew W Moskewicz, Khalid Ashraf, William J Dally, and Kurt Keutzer, "Squeezenet: Alexnet-level accuracy with 50x fewer parameters andi $0.5 \mathrm{mb}$ model size," arXiv preprint arXiv:1602.07360, 2016. 